\title{
Information-Centric Networking (ICN) Architectures for Integration of Satellites into the Future Internet
}

\author{
Vasilios A. Siris, Christopher N. Ververidis, George C. Polyzos, and Konstantinos P. Liolis
}

\begin{abstract}
This paper discusses the integration of satellite and terrestrial networks over an Information Centric Networking (ICN) architecture. Focus is given on the identification of the key features that characterize ICN architectures and how these may impact, but also be affected by, the integration of satellite and terrestrial networks. The work presented contains intermediate results obtained as part of the ongoing ESA ARTES study ' $\varphi \mathrm{SAT}$ ' on the role of satellites in the Future Internet.
\end{abstract}

Index Terms-Information-Centric Networking (ICN), Future Internet (FI), Satellite-Terrestrial Networks Integration

\section{INTRODUCTION}

$\mathrm{I}^{\mathrm{N}}$ $\mathrm{N}$ the last few years there is pressure on the current Internet architecture to meet new and emerging needs of its users. Inefficiencies of the current Internet architecture with regard to, e.g., mobility support, traffic management, or content delivery, have been highlighted along with the complexities of proposed work-arounds or patches, which have progressively led to the ossification of the Internet. The root of these inefficiencies is the fact that the current Internet's host-centric communication model does not match the Internet's dominant usage, which involves end-users exchanging information or accessing services, independent of the device where the information is located or which provides the service.

Under this pressure, many research initiatives have started to investigate Information-Centric Networking (ICN) as the fundamental paradigm for the Future Internet. ICN architectures decouple the data (service) from the actual devices storing (providing) it through location-independent naming. This decoupling allows tackling problems that

This work is being done under the ESA ARTES 1 project $\varphi$ SAT: The Role of Satellite in Future Internet Services (ESA/ESTEC Contract No.: $4000103360 / 11 / \mathrm{NL} / \mathrm{NR})$. Responsibility for the presented content resides with the authors.

V. A. Siris is with the Mobile Multimedia Laboratory, Department of Informatics, Athens University of Economics and Business, Greece (phone: +302108203 581; fax: +302108226 105; e-mail: vsiris@aueb.gr).

C. N. Ververidis is with the Mobile Multimedia Laboratory, Department of Informatics, Athens University of Economics and Business, Greece (e-mail: chris@aueb.gr).

G. C. Polyzos is with the Mobile Multimedia Laboratory, Department of Informatics, Athens University of Economics and Business, Greece (e-mail: polyzos@aueb.gr).

K. P. Liolis is with the IT, Applications and R\&D Division, Space Hellas S.A., Athens, Greece (e-mail: klio@space.gr). emerge with host mobility much more efficiently, since now the identification of information/content (which remains the same irrespectively of the location of its provider/owner) takes the position of the identification of communication end-points (which may change or move). The identification of content at the network layer facilitates data caching in network elements (in-network caching) and more efficient content delivery without resorting to add-on, often proprietary and costly overlay solutions (e.g., CDNs). Location-independent naming also facilitates information collection or the retrieval of different information segments belonging to the same file from multiple sources, without requesting information from each source individually. Moreover, naming of content facilitates data collection and data dissemination supporting many/any-to-one, one-to-many/any, and many/any-tomany/any deliver modes. Such delivery modes are the basis for smart transport and energy systems, machine-to-machine communications, and the Internet of things. Satellite networks can augment these capabilities with their wide-area coverage and inherent broadcast capabilities.

ICN additionally promotes a publish/subscribe information model where receivers will not receive information unless they have explicitly requested or subscribed for it, thus making the architecture more robust against DoS (Denial of Service) attacks. ICN's resolution service is responsible for locating the desired content, by matching information requests to publishers where the content is available. After resolution, the routing and forwarding functions transfer information from the publishers to the subscribers (receivers).

Future Internet ICN-related research efforts have thus far focused solely on terrestrial networks, neglecting the opportunity of integrating satellite and terrestrial networks by using a common ICN architecture that combines and exploits the advantages of both networks. To the best of our knowledge, this is the first paper that discusses features of various ICN architectures for the Future Internet (FI) and their implications and the corresponding advantages, disadvantages, and tradeoffs when they are applied for the integration of satellite and terrestrial networks. The paper [17] builds upon the results presented hereinafter, focusing on the satelliteterrestrial network integration scenarios matching the FI ICNrelated concepts.

The rest of this paper is organized as follows: In Section II we identify and discuss key features of ICN architectures, 
which include mobility support, in-network caching, contentaware traffic management, degree of coupling between resolution and data transport, degree of coupling between data routing (topology management) and forwarding, and transport and congestion control. In Section III we discuss the implications of these features and how advantages of satellite networks, such as wide-coverage and inherent broadcast support, can be exploited and further motivate the adoption of ICN architectures for integrating satellite and terrestrial networks. We also discuss how features of ICN architectures can help address issues in satellite networks, such as long propagation delay and varying network topology in LEO constellations. Finally, in Section IV we conclude the paper.

\section{KEY FEATURES OF ICN ARCHITECTURES}

The Information-Centric Networking (ICN) concept has been proposed to address the new requirements imposed by modern applications as well as the tremendous growth of mobile and wireless computing. Rather than assigning unique addresses to end-hosts connected by communication links, as in the current Internet, ICN architectures assign unique names to information objects (content) and utilize the publishsubscribe model for information transfer. According to the publish-subscribe communication model as implemented in ICN, end-hosts advertise their interest (subscription) in receiving information objects to the network but also their ability to provide specific information objects (publication). By utilizing the publish-subscribe communication model, ICN shifts the power from the sender of information to the receiver in the sense that information will be delivered to an end-host if and only if it has previously declared interest in receiving this information. The network takes up the role of matching interests (or subscriptions) to publications (information objects); this is commonly referred to as the resolution or rendezvous function. Node or link identifiers of course are not necessarily eliminated in the sense that they can be needed for lower level topology maintenance mechanisms and for associating nodes with the content they provide. However, the core idea is that the content is identified, addressed, and matched independently of its location (location-identity split). Resolution can be performed by a separate name resolution system (Figure 1), which comprises of interconnected name resolution servers.

In addition to name resolution, the other two key functions of ICN architectures are routing (or topology management) and forwarding. Routing involves determining a path from the publisher to the subscriber, based on a subscription and publication match provided by the name resolution system. Forwarding involves moving information from the publishers to the subscriber along the determined path. Routing and forwarding can be implemented in a coupled hop-by-hop fashion, as is currently performed by IP, or can be performed separately (decoupled). We discuss this in more detail later.

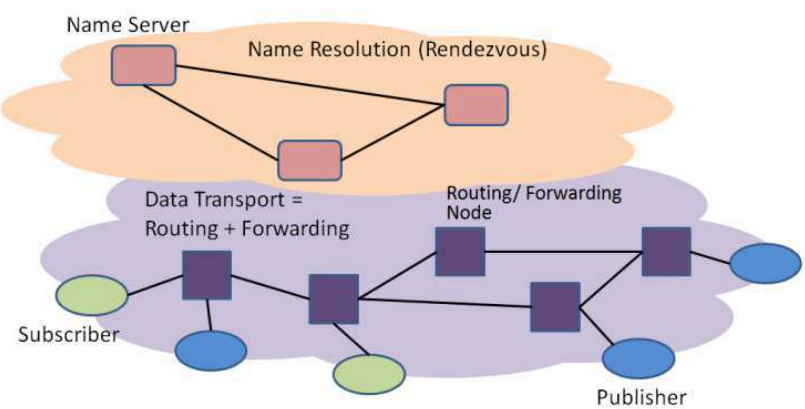

Figure 1: Three key functions of ICN architectures are name resolution (or rendezvous), routing (or topology management), and forwarding.

Many ICN architectures have been proposed so far in the framework of research projects including Publish-Subscribe Internet Technology (PURSUIT) [1] and its predecessor Publish-Subscribe Internet Routing Paradigm (PSIRP) [2], COntent Mediator architecture for content aware nETworks (COMET) [4], CONVERGENCE [5], 4WARD [6] and its successor project Scalable \& Adaptive Internet soLutions (SAIL) [7], DONA [8], and NDN [9], its predecessor CCN and ANR Connect [10], a project that focuses on the $\mathrm{CCN} / \mathrm{NDN}$ architecture. The key features that characterize all ICN architectures are mobility support, in-network caching, content-aware traffic management, degree of coupling between resolution and data transport, degree of coupling between data routing (topology management) and forwarding, and transport and congestion control. In the following paragraphs we discuss each of these features.

\section{A. Mobility Support}

ICN architectures promote a receiver-driven information request model, where nodes receive only the information which they have requested or subscribed to. This is in contrast to the current Internet's model where the sender has full control of the data he/she can send. Additionally, ICN's request model and content transfer from sources to receivers is connectionless, in contrast to TCP's connection-oriented (statefull) end-to-end control that involves location-dependent addresses. Both the above features allow mobiles that have changed their position (network attachment point) to simply re-issue requests for information objects they didn't receive while they were connected to their previous attachment point or while they were disconnected. Hence, delay/disruption tolerant operation in addition to mobility is supported without requiring cumbersome solutions such as mobile IP.

Specific schemes for enhancing mobility support have also been proposed in the context of ICN architectures proposals. In rendezvous based schemes the rendezvous service has the major role. The (moving) subscriber upon re-location and reattachment to the network needs to re-issue a subscription for the content he/she did not receive due to their movement. Upon receipt of this subscription the rendezvous service returns the new path for connecting the subscriber with a 
publisher (either the same or a new one). Depending on the service (streaming or file transfer), lost packets (those that were being transferred during the handoff) may need to be recovered or not. If packets need to be recovered then the new subscription may also contain a hint about the last successfully received chunk of content, which the rendezvous service communicates to the newly chosen publisher, so that lost packets can be recovered. If it is useless to recover packets (e.g., if the subscription is for a realtime video stream and the playout time for the frames contained in the lost packets has passed) then the rendezvous service simply returns the new path from the publisher to the re-located subscriber. Some approaches (like $\mathrm{CCN}$ ) however require that subscriptions (or interests) have to be issued for every packet, so in that case the subscriber upon re-attaching to a new location simply reissues the non-satisfied interests. In the case of publisher (or source) mobility, the publisher needs to notify the rendezvous service about its relocation, such that new paths can be established with the subscribers that this particular publisher had been serving before moving. However, in case that there are multiple publishers offering the same content with the publisher that moved, the rendezvous service may also choose to assign some (or all) of the subscribers that were served by the publisher which moved to other publishers (e.g., to publishers that are now located closer to these subscribers). Other schemes, such as [11] and [12], manipulate naming in order to introduce topological hints in subscription and publication identifiers and also try to localize mobility management signaling.

\section{B. In-network caching}

Through location-independent naming of information objects, ICN architectures can support in-network caching in a seamless manner. In this sense network elements do not see opaque IP packets but pieces of content which can be cached and subsequently delivered to requestors irrespective of whether the original information publisher is still accessible or not (time decoupling between information publishers and requestors). Additionally, by naming individual chunks or packets of an information object, caching can be performed at a fine granularity allowing more efficient utilization of buffers and multiple network paths for content delivery.

There are mainly two distinct types of caching [3]: on-path caching and off-path caching. With on-path caching a subscriber can be served by a cache located on the path followed by the request while it is being routed towards the data source. Off-path caching on the other hand, refers to the system's ability of serving item requests by means of caching points that do not lie on the path between the requestor and the originating server, thus utilizing available storage in the network. The difference between these two types of caching schemes is that on-path caching is transparent to the resolution system, while off-path caching requires caches to inform the resolution system about the content they store. Off-path caches are handled by the name resolution system in the same way as publishers of information.

\section{Content-aware traffic management}

By exposing the content name and type to the network/forwarding layer, content-aware traffic management, prioritization, and QoS support can be readily applied, without requiring add-on hardware and costly mechanisms, such as deep packet inspection. Optimizations that target at the same time the selection of the best provider (publisher) of a given information object (or providers in case of content delivery from multiple sources) and the formation of optimal (multicast) delivery trees over the most appropriate routing paths (less congested, lowest delay etc.) can now be performed at the network layer.

\section{Different degrees of coupling of resolution and data transport}

Name resolution and data transport in ICN can be fully coupled, in which case requests for information objects are routed in the network until the corresponding information objects are found, and subsequently transferred to the requesting nodes using the reverse of the path followed by the requests. This is the approach followed by CCN/NDN, where requesting nodes issue Interests, which are registered in the Pending Interest Table (PIT) of routing elements along with the interface they arrived from. An Interest is propagated based on the Forward Information Base (FIB) table until it reaches a data source that can satisfy the request. The source sends the Data packet that follows (based on the PITs of network elements) the inverse of the path that the Interest packet has travelled. Moreover, each network element along the path erases the corresponding entry from its PIT (duplicate Interest packets are dropped when no corresponding PIT entry is found).

Alternatively, resolution can be handled by a separate service that matches subscriptions for information objects with publications for information objects, which is independent of the data transport functionality. This approach, which is followed by PSIRP/PURSUIT, introduces flexibility in the implementation and management of these network functions.

\section{E. Different degrees of coupling of data routing (topology management) and forwarding}

Routing and forwarding can be coupled, as in the current IP protocol, or can be decoupled, in which case route selection is performed independently, and data forwarding is performed using, e.g., label switching/forwarding. Moreover, the decoupled approach allows different forwarding mechanisms to be used in different (possibly heterogeneous) networks (or network segments) while using a common routing mechanism.

Routing and forwarding is an area where ICN architectures show major differences. DONA [8] and CURLING [4] work over IP and thus keep intact the current routing and forwarding functionality. In contrast, PSIRP/PURSUIT [1][2], 4WARD/SAIL [6][7] and CCN/NDN [9] bring significant changes to the routing and forwarding model. The first two architectures assume the operation of topology discovery protocols (e.g., a link-state routing protocol) for collecting 
topology information, but employ source routing techniques for avoiding state maintenance in routers. In theses approaches forwarding is done on a hop-by-hop basis depending on the path that is encoded in the packet header either as a Bloom Filter in the case of PURSUIT [13] or as a Compact Identifier in 4WARD/SAIL [14]. Hence routing and forwarding is clearly separated in these architectures. In contrast, in CCN/NDN (also in CONVERGENCE which operates on top of $\mathrm{CCN} / \mathrm{NDN}$ ) forwarding of Data packets are based on their names and PIT table entries, which create a path that is the inverse of the path followed by Interest packets towards a source that can satisfy them.

\section{F. Transport and congestion control}

ICN architectures promote hop-by-hop or segment-bysegment congestion control, which departs from TCP's endto-end control model. Such an approach can better accommodate links with long delays and disruptions. It further allows effective control of traffic that has to go through multiple networks (or network segments) with different physical layer characteristics. Moreover, the integration of caching and replication deep in the network allows ICN architectures to optimize the transport layer functionality. Delivery modes such as multicast (i.e., one-to-many) and concast (many-to-one), the ability of the network to apply anycast, as well as the support for multi-path routing in several ICN approaches, offer a rich set of mechanisms affecting the design of flow, congestion, and error control functions.

\section{IMPLICATIONS OF ICN TO THE INTEGRATION OF SATELlite AND TERRESTRIAL NETWORKS}

The previous section described the main functionalities of ICN architectures as well as their key features. In this section we discuss the possible implications of these features on the integration of satellite and terrestrial networks. Our focus is on how the advantages of satellite networks, namely wide-area coverage and inherent broadcast/multicast support, can be exploited by ICN architectures. This can motivate the integration of satellite and terrestrial networks using an ICN architecture. Additionally, we discuss how important issues of satellite networks, which include high propagation delay and varying network topology in the case of LEO satellite networks, can be addressed through capabilities of ICN architectures. Finally, we discuss where and how satellite capabilities such as On-Board Processing (OBP) can be exploited in an integrated satellite-terrestrial architecture.

\section{A. Mobility Support}

ICN's receiver-driven and connectionless information request model, in addition to end-station mobility, can facilitate mobility due to changing network topology, such as in the case of LEO satellite constellations, avoiding the need for complex inter-satellite routing control protocols and handovers.

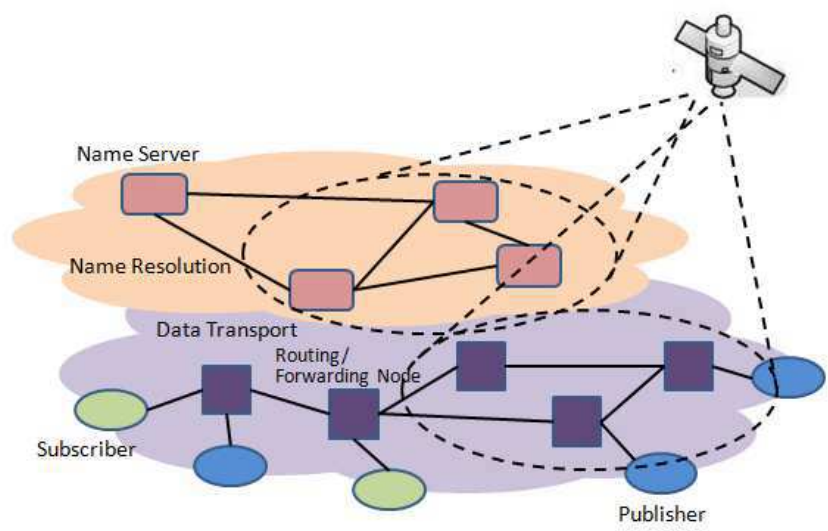

Figure 2: A satellite's wide-coverage and broadcast support can impact both resolution and data transport (routing and forwarding).

Unlike receiver mobility, which is inherently supported by ICN architectures, support for publisher (source) mobility requires updating of routing or name resolution tables. The wide-coverage and native broadcasting capabilities of satellite networks can significantly reduce the overhead and delay for such updates, compared to terrestrial-only networks. In particular, in $\mathrm{CCN}$ networks information requests (Interests) are routed based on Forward Information Base (FIB) tables. FIB tables will need to be updated in the case of publisher mobility. On the other hand, in ICN architectures where name resolution, topology control, and forwarding are separated, such as in the PSIRP/PURSUIT architecture, publisher mobility can result a) in changes in routing tables, if changes of a publisher's location results in different paths being optimal or preferred, and b) in changes in forwarding tables, in order to ensure continuous connectivity of ongoing connections. Moreover, if publisher mobility results in changes of the content different servers can provide, resolution tables, based on which information request and publication matching is performed, need to be updated.

\section{B. In-network caching}

Seamless support for in-network caching can help reduce the negative impact of long propagation delays of satellite links, by caching content close to users before they request it. In this direction, the wide-area coverage and native broadcasting of satellite networks can be used to simultaneously update multiple caches without additional cost and with low delay. Also, the finer granularity and ubiquitous support for caching, together with content-awareness through naming of the content, can facilitate more efficient utilization of caches, which is especially important if caches are located on satellites.

As discussed in the previous section, we can differentiate between two types of in-network caches: on-path and off-path caches. On (Off)-path refers to whether the cache is in (is not in) the path followed by the information requests. On-path caches can be readily checked if they contain content that satisfies a request. On the other hand, off-path caches can be exploited only if there exists a discrete name resolution system 
that has knowledge of their existence and of the contents they can provide. In the case of satellite networks, support for onpath on-satellite caches can be more difficult and costly, whereas support for on-path caches located in satellite gateways and on-path caches located in ground nodes is easier. An investigation of caching for information-centric satellite networks is contained in [15].

\section{Content-aware traffic management}

Content-aware traffic management can be utilized in the case of satellites with on-board processing capabilities, in order to provide content-aware prioritization and QoS support, while efficiently utilize costly satellite capacity. Moreover depending on the specific requirements of flows, decisions can be taken on whether to route flows through the satellite or the terrestrial network, hence to exploit multipath support in a content-aware manner.

Additionally, content-awareness can help to more effectively exploit the wide-coverage broadcast/multicast capability of satellite networks, by deciding which flows to broadcast/multicast based directly on the content they carry. It is important to note here that content-aware traffic prioritization, QoS support, and broadcasting/multicasting should be addressed in combination to content-aware innetwork cache management and content replication, since the combined behavior of these functions affects the overall performance that end-users experience.

\section{Different degrees of coupling of resolution and data transport}

Decoupling of name resolution (or rendezvous) and data transport allows different entities to implement each of these functionalities. This can be desirable from a socio-economic perspective, since it helps to define - at an architecture level clear boundaries between modules and entities implementing different functionalities. Such clear boundaries are important in order to address "tussles", i.e., conflicts of interests between different stakeholders in the Internet [16].

Decoupling of name resolution and data transport can also facilitate support for multiple naming systems over the same data transport infrastructure or the integration of infrastructures with a different naming system. This can be important in the case of satellite networks, which up to now have been primarily used for video and TV broadcasting.

Name resolution involves the exchange of control traffic (information requests). Coupling of name resolution and data transport results in the data following the reverse of the path that is followed by the information requests. Such a coupling between information request and data paths can assist in the exploitation of on-path caches with purely local mechanisms. On the other hand, decoupling name resolution and data transport enables usage of different paths for control traffic and for data traffic. This can be beneficial for the integration of satellite networks, which typically involve high propagation delays, with terrestrial networks: Information requests (control traffic) can utilize low latency terrestrial links, while data transport can utilize wide coverage and high capacity satellite links. As an example, video streaming can be performed over satellite links while video playback control can be sent through the terrestrial network.

TABLE I

ISSUES FOR THE INTEGRATED NETWORK AND SOLUTIONS THROUGH ICN ARCHITECTURE

\begin{tabular}{|c|c|}
\hline $\begin{array}{c}\text { Issues for the integrated } \\
\text { satellite-terrestrial network }\end{array}$ & $\begin{array}{c}\text { Solutions through an ICN } \\
\text { architecture }\end{array}$ \\
\hline $\begin{array}{l}\text { High propagation delay at the } \\
\text { satellite segment }\end{array}$ & $\begin{array}{l}\text { Highly efficient and ubiquitous in- } \\
\text { network caching }\end{array}$ \\
\hline $\begin{array}{l}\text { Terrestrial segment more } \\
\text { appropriate for low delay } \\
\text { interactive services }\end{array}$ & $\begin{array}{l}\text { Multipath mechanisms and content- } \\
\text { based routing allows different types of } \\
\text { traffic to be routed through different } \\
\text { networks, and allows the separation of } \\
\text { control and data paths }\end{array}$ \\
\hline $\begin{array}{l}\text { Heterogeneity of physical } \\
\text { layer characteristics across } \\
\text { segments of the end-to-end } \\
\text { path that spans the integrated } \\
\text { satellite-terrestrial network }\end{array}$ & $\begin{array}{l}\text { Separation of routing and forwarding } \\
\text { allows different forwarding } \\
\text { mechanisms to be applied in different } \\
\text { network segments and domains based } \\
\text { on their particular characteristics and } \\
\text { requirements }\end{array}$ \\
\hline & $\begin{array}{l}\text { Hop-by-hop congestion control can } \\
\text { accommodate the different } \\
\text { characteristics of satellite links, such } \\
\text { as long propagation delays and losses }\end{array}$ \\
\hline $\begin{array}{l}\text { Changing network topology } \\
\text { in the case of LEO satellite } \\
\text { constellations }\end{array}$ & $\begin{array}{l}\text { Receiver-driven request model and } \\
\text { connectionless transport can jointly } \\
\text { support seamless connectivity } \\
\text { together with delay/disruption } \\
\text { tolerance }\end{array}$ \\
\hline $\begin{array}{l}\text { Satellite segment appropriate } \\
\text { for data broadcasting/ } \\
\text { multicasting services }\end{array}$ & $\begin{array}{l}\text { Satellite's wide coverage and inherent } \\
\text { broadcast/multicast capabilities can be } \\
\text { combined with ICN's content-aware } \\
\text { data collection and dissemination, } \\
\text { further improving its gains in this } \\
\text { direction }\end{array}$ \\
\hline $\begin{array}{l}\text { Complexity and cost in } \\
\text { adding functionality on-board } \\
\text { satellites }\end{array}$ & $\begin{array}{l}\text { Decoupling of the key ICN functions } \\
\text { (resolution, routing, and forwarding) } \\
\text { can allow different entities to } \\
\text { implement individual functionalities, } \\
\text { hence the complexity and cost of } \\
\text { satellite nodes can be reduced by } \\
\text { having them implement only } \\
\text { forwarding }\end{array}$ \\
\hline $\begin{array}{l}\text { Management of costly } \\
\text { satellite capacity }\end{array}$ & $\begin{array}{l}\text { Content-aware traffic management } \\
\text { can allow flexible utilization of } \\
\text { satellite capacity, jointly utilizing } \\
\text { mechanisms such as wide-area } \\
\text { broadcasting and in-network caching }\end{array}$ \\
\hline $\begin{array}{l}\text { Satellite Gateway (SG) nodes } \\
\text { have a key role for } \\
\text { interconnecting satellite and } \\
\text { terrestrial networks }\end{array}$ & $\begin{array}{l}\text { SG nodes are ideal to implement } \\
\text { rendezvous and caching } \\
\text { functionalities for integrating satellite } \\
\text { and terrestrial networks } \\
\text { SG nodes can implement mapping and } \\
\text { conversion functions to support } \\
\text { interworking of different forwarding } \\
\text { mechanisms in satellite and terrestrial } \\
\text { networks }\end{array}$ \\
\hline
\end{tabular}

It is important to note that there exist solutions that separate control and data traffic over satellite and terrestrial networks. However, these solutions are implemented at the application layer and hence are application specific. On the contrary, support for multiple paths and selection based on content is inherently supported in ICN networks, hence is application 
independent. Thus, a disadvantage of satellite networks (high propagation delay) can be addressed while exploiting a key advantage of satellite networks (wide coverage and high bandwidth).

Decoupling of resolution and data transport also allows more flexibility for applying access and usage control rules and QoS policies that can efficiently utilize satellite and terrestrial resources in a unified manner. For example, the resolution function can focus on implementing access and usage control rules, whereas the data transport (routing and forwarding) functionality can focus on application and content specific QoS policies.

\section{E. Different degrees of coupling of data routing (topology management) and forwarding}

Decoupling of routing (topology management) and forwarding can simplify the integration of satellite and terrestrial networks and the deployment of ICN, since the forwarding mechanisms that currently exist in satellite networks can be used without modification. The interworking of different forwarding mechanisms in satellite and terrestrial networks can be addressed through mapping and conversion functions in satellite gateways. In general, separation of the resolution, routing, and forwarding functions provides higher flexibility of where these mechanisms can be implemented, such as in satellites with on-board processing capabilities, terrestrial-satellite gateways, and ground stations, and leads to clear boundaries that are important to address tussles between different stakeholders with conflicting interests, as discussed in the previous subsection. Moreover, decoupling allows easier exploitation of multiple paths from a publisher to a subscriber, in order to improve resilience, throughput, and load balancing.

On the other hand, coupling between routing and forwarding requires that both functionalities are implemented in all network nodes, thus resulting in more complex and costly nodes. However, coupling between routing and forwarding can make it easier to overcome short-lived link disruptions through local re-routing actions.

\section{F. Transport and congestion control}

Hop-by-hop or segment-by-segment congestion control that is promoted by ICN architectures can better address the long propagation delays of satellite links, providing a unifying framework for delay tolerant networking, while avoiding overlay solutions such as performance enhancing proxies.

Moreover, hop-by-hop control can help exploit multipath and multisource transport, which yields more efficient utilization of costly satellite resources, and can help adaptation to accommodate a dynamic network topology, which is the case of LEO satellites constellations.

\section{CONCLUSION}

This paper has discussed key features of ICN architectures and their implications for the integration of satellites in the Future Internet. The features include mobility support, innetwork caching, content-aware traffic management, degree of coupling between resolution and data transport, degree of coupling between data routing (topology management) and forwarding, and transport and congestion control. Our goal was to discuss how main advantages of satellite networks, namely wide-area coverage and inherent broadcast support, can be exploited to increase the gains in adopting ICN architectures for integrating satellite and terrestrial networks, and to discuss how capabilities of ICN architectures can address key issues in satellite networks, such as long propagation delays and varying network topology in the case of LEO satellite constellations. The key points from the above discussion are summarized in Table I.

Our ongoing work includes validating the advantages identified in this paper, based on the satellite-terrestrial Future Internet network integration scenarios identified in [17].

\section{ACKNOWLEDGMENT}

The authors would like to thank Dr. Maria Guta from the European Space Agency (ESA/ESTEC) for her valuable comments during the preparation of this paper.

\section{REFERENCES}

[1] PURSUIT Project, http://www.fp7-pursuit.eu

[2] PSIRP Project - Deliverable D4.6: Final Evaluation Report on Deployment Incentives and Business Models, Available at http://www.psirp.org/publications/

[3] G. Xylomenos, X. Vasilakos, C. Tsilopoulos, V.A. Siris and G.C.Polyzos, "Caching and Mobility Support in a Publish-Subscribe Internet Architecture," IEEE Communications Magazine, vol. 50, no. 7, pp. 52-58, Jul. 2012.

[4] W. Koong Chai et al., "CURLING: Content-Ubiquitous Resolution and Delivery Infrastructure for Next-Generation Services," IEEE Communications Magazine, vol. 49, no. 3, pp. 112-120, Mar. 2011.

[5] CONVERGENCE Project, http://www.ict-convergence.eu/

[6] B. Ahlgren, P.A. Aranda, P. Chemouil, S. Oueslati, L.M. Correia, H. Karl, M. Sollner, and A. Welin, "Content, Connectivity, and Cloud: Ingredients for the Network of the Future," IEEE Communications Magazine, vol. 49, no. 7, pp. 62-70, Jul. 2011.

[7] SAIL Project, http://www.sail-project.eu/

[8] T. Koponen, M. Chawla, B. Chun, A. Ermolinskiy, K. H. Kim, S. Shenker, and I. Stoica. "A data-oriented (and beyond) network architecture," ACM SIGCOMM Computer Communication Review, vol. 37, no. 4, pp. 181-192, Aug. 2007.

[9] Named Data Networking project, http://www.named-data.net/

[10] ANR Connect Project, http://anr-connect.org/

[11] Ravishankar Ravindran, Samantha Lo, Xinwen Zhang, and Guoqiang Wang, "Supporting Seamless Mobility in Named-Data Networking," Proc. 5th International Workshop on the Network of the Future (colocated with ICC'12), 2012.

[12] 4WARD Project Deliverable D-6.1 First NetInf Architecture Description: http://www.4ward-project.eu/

[13] P. Jokela, A. Zahemszky, C. E. Rothenberg, S. Arianfar, and P. Nikander, "LIPSIN: line speed publish/subscribe internetworking," Proc. ACM SIGCOMM 2009.

[14] SAIL Project Deliverable D-3.1 (D-B.1) The Network of Information: Architecture and Application: http://www.sailproject.eu/wpcontent/uploads/2011/08/SAIL_DB1_v1_0_final-Public.pdf

[15] L. Galluccio, G. Morabito, and S. Palazzo, "Caching in InformationCentric Satellite Networks," Proc. ICC 2012.

[16] D. D. Clark, J. Wroclawski, K. R. Sollins, and R. Braden, "Tussle in Cyberspace: Defining Tomorrow's Internet," IEEE/ACM Transactions on Networking, vol. 13, no. 3, pp. 462-475, Jun. 2005.

[17] K.P. Liolis, A. Drougas, I.A. Andrikopoulos, F. Arnal, C. Baudoin, C.N. Ververidis, V.A. Siris, G.C. Polyzos, and M. Guta, " Satellite-Terrestrial Integration Scenarios for Future Information-Centric Networks", in Proc. 30th AIAA International Communications Satellite Systems Conference (ICSSC 2012), Ottawa, Canada, Sep. 2012. 